routes (horizontal vs. perinatal transmission) reflecting duration of infection might be reasons when compared with an undetectable HBV DNA rate of $100 \%$ in patients from Argentina and Europe. ${ }^{2-4}$ However, these differences cannot explain the much higher 3-year $\mathrm{HBeAg}$ seroconversion rate (53\%) and undetectable HBV DNA rate (96\%) in 222 patients from Hong Kong, ${ }^{5}$ 3-year undetectable $\mathrm{HBV}$ DNA rate of $93 \%$ (89\% in $\mathrm{HBeAg}$ positive, $100 \%$ in $\mathrm{HBeAg}$ negative) in 474 Japanese patients ${ }^{6}$ and an undetectable HBV DNA rate of $96.6 \%$ in 381 HBeAg-negative Taiwanese patients with compensated liver cirrhosis. ${ }^{7}$ In contrast to studies of single or three university centres, ${ }^{5-7}$ their patients came from 26 community or university centres with great variations in HBV DNA and ALT ranges at each laboratories, ${ }^{1}$ their lower response rates may therefore reflect the more 'real' real-world results.

(ii) Sixteen per cent of their patients discontinued therapy, 36 by provider recommendation and 48 by themselves or lost to follow-up. It was inferred that self-discontinuation of therapy might encounter unpredictable untoward outcomes because of inadequate offtherapy monitoring. ${ }^{8}$ It would be more informative to confirm or refute this inference if the authors could provide more information to compare the outcomes between the patients who stopped entecavir therapy by themselves and those by provider recommendation.

(iii) Four (6.6\%) of their 61 patients with cirrhosis developed new hepatic decompensation after initiation of entecavir. This is surprisingly high when compared with none of the 204 Italian patients with cirrhosis treated with entecavir for 5 years. ${ }^{2}$ It would be also informative to know what happened with these patients.

Taken together, this study disclosed a lower response rate and a high self-discontinuation rate during a median of 4 years. This is indeed a 'real-world' challenge in maintaining indefinite long-term/lifelong therapy. A recent systematic review in this journal suggests that

\section{Letter: lessons from the 'real-world' entecavir therapy in chronic hepatitis B patients - authors' reply}

\footnotetext{
J. Ahn*, M. H. Nguyen ${ }^{\dagger}$, J. K. Lim*, H. M. Lee ${ }^{\S}$, C. Q. $\operatorname{Pan}^{\Uparrow} \&$ A. S. Lok ${ }^{\star \star}$

*Division of Gastroenterology \& Hepatology, Oregon Health \& Science University, Portland, OR, USA.

†Division of Gastroenterology \& Hepatology, Stanford University, Stanford, CA, USA.
}

cessation of long-term anti-viral therapy prior to HBsAg loss/seroclearance is a safe and feasible alternative to indefinite treatment if an appropriate stopping rule and a proper off-therapy monitoring plan are provided for timely retreatment. ${ }^{8}$ Perhaps this 'stop and watch' strategy is safer than self-discontinuation, and therefore it needs to be considered in guidelines of major liver associations.

\section{ACKNOWLEDGEMENTS}

Declaration of personal interests: YF Liaw has involved in clinical trials or served as a global advisory board member of Roche. Declaration of funding interests: None.

\section{REFERENCES}

1. Ahn J, Lee HM, Lim JK, et al. Entecavir safety and effectiveness in a national cohort of treatment-naive chronic hepatitis B patients in the US - the ENUMERATE study. Aliment Pharmacol Ther 2016; 43: 134-44.

2. Lampertico P, Vigano M, Soffredini R. Entecavir monotherapy for nuc-naieve chronic hepatitis $B$ patients from field practice: high efficacy and favorable safety profile over 3 year. Hepatology 2011; 54: A1436.

3. Zoutendijk R, Reijnders JG, Zoulim F, et al. Virological response to entecavir is associated with a better clinical outcome in chronic hepatitis B patients with cirrhosis. Gut 2013; 62: 760-5.

4. Ridruejo E, Marciano S, Galdame O, et al. Efficacy and safety of long term entecavir in chronic hepatitis B treatment naive patients in clinical practice. Ann Hepatol 2014; 13: 327-36.

5. Yuen MF, Seto WK, Fung J, et al. Three years of continuous entecavir therapy in treatment-naive chronichepatitis B patients: VIRAL suppression, viral resistance, and clinical safety. Am J Gastroenterol 2011; 106: 1264-71.

6. Ono A, Suzuki F, Kawamura Y, et al. Long-term continuous entecavir therapy in nucleos( $\mathrm{t}$ )ide-naive chronic hepatitis B patients. J Hepatol 2012; 57: 508-14.

7. Chen YC, Peng CY, Jeng WJ, Chien RN, Liaw YF. Clinical outcomes after interruption of entecavir therapy in HBeAgnegative chronic hepatitis B patients with compensated cirrhosis. Aliment Pharmacol Ther 2015; 42: 1182-91.

8. Chang ML, Liaw YF, Hadziyannis SJ. Systematic review: cessation of long-term nucleos(t)ide analogue therapy in patients with hepatitis B e antigen-negative chronic hepatitis B. Aliment Pharmacol Ther 2015; 42: 243-57.

"Digestive Diseases, Yale University, New Haven, CT, USA.

${ }^{\S}$ Gastroenterology/Hepatology Division, Tufts Medical Center, Boston, MA, USA.

"Department of Medicine, NYU Langone, New York, NY, USA

${ }^{\star *}$ Division of Gastroenterology and Hepatology, University of

Michigan, Ann Arbor, MI, USA.

E-mail: ahnj@ohsu.edu

doi:10.1111/apt.13558 
SIRS, We appreciate the interest and comments from Drs Liaw and Chang on our recent report on the safety and effectiveness of entecavir in 'real-world' patients with chronic hepatitis B in the United States (USA). ${ }^{1,2}$ The comments highlighted the impact and importance of 'real-world' challenges in maintaining long-term therapy in patients with chronic hepatitis B infection.

As they pointed out, our study had lower 3-year HBV DNA complete suppression rates and 5-year HBeAg seroconversion rates than reported in previous international studies from Argentina, Europe and Asia. ${ }^{3,} 4$ The majority of our patients were also Asian (83\%), and 61\% were born outside the USA. Thus, demographic or presumed transmission route differences cannot account fully for the differences in virological outcomes from the Asian studies, though they may provide some basis for the observed differences from the Argentinian and European studies. The more likely reason for our lower response rates, as also suggested by Drs Liaw and Chang, is the 'real-world' outcomes in this US study of 26 individual sites and their associated heterogeneity in patient population, provider practices and laboratory testing. Lower HBeAg seroconversion rates have also been reported by a number of smaller studies from the USA and elsewhere. ${ }^{5}$

Of the four patients with cirrhosis who developed new hepatic decompensation while on entecavir, three were alive at last follow-up and one had died after being listed for liver transplantation. The relatively small number of patients with cirrhosis $(n=66)$ limits our ability to generalise our findings regarding hepatic decompensation while on entecavir therapy. Furthermore, we do not have data whether these four patients had other causes of liver disease such as alcohol or non-alcoholic steatohepatitis.

With regard to the comparison of patients who self-discontinued therapy vs. those who did so by provider recommendation, reliable comparison of outcomes cannot be made as patients who self-discontinued were generally lost to follow-up or lacked sufficient follow-up data due to the lack of regular monitoring. Therefore, we cannot confirm the concern that there could be worse outcomes for those who self-discontinued entecavir vs. those whose entecavir was stopped by their providers. However, we fully agree that maintenance of adherence to long-term treatment is critical but remains a challenge in 'real-world' practice, as previously discussed in our paper. Furthermore, we concur that strategies to maintain adherence and to identify those patients who may be eligible for a 'stop and watch' approach, to reduce the risk of self-discontinuation, will also be safer and critical to optimise 'real-world' treatment outcomes in patients with chronic hepatitis B infection.

\section{ACKNOWLEDGEMENT}

The authors' declarations of personal and financial interests are unchanged from those in the original article. ${ }^{2}$

\section{REFERENCES}

1. Chang M-L, Liaw Y-F. Letter: lessons from the "real-world" entecavir therapy in chronic hepatitis B patients. Aliment Pharmacol Ther 2016; 43: 846-7.

2. Ahn J, Lee HM, Lim JK, et al. Entecavir safety and effectiveness in a national cohort of treatment-naive chronic hepatitis B patients in the US - the ENUMERATE study. Aliment Pharmacol Ther 2016; 43: 134-44.

3. Zoutendijk R, Reijnders JG, Zoulim F, et al. Virological response to entecavir is associated with a better clinical outcome in chronic hepatitis B patients with cirrhosis. Gut 2013; 62: 760-5.

4. Yuen MF, Seto WK, Fung J, et al. Three years of continuous entecavir therapy in treatment-naive chronic hepatitis B patients: VIRAL suppression, viral resistance, and clinical safety. Am J Gastroenterol 2011; 106: 1264-71.

5. Liu A, Ha N, Lin B, et al. Low hepatitis B envelope antigen seroconversion rate in chronic hepatitis $\mathrm{B}$ patients on long-term entecavir $0.5 \mathrm{mg}$ daily in routine clinical practice. Eur J Gastroenterol Hepatol 2013; 25: 338-43.

\section{Letter: orlistat as a potential treatment for chronic idiopathic constipation}

\author{
F. Iqbal ${ }^{\star}$, M. Samuel ${ }^{\star}$, E. J. K. Tan ${ }^{\dagger}$, R. J. Nicholls* \& \\ C. J. Vaizey ${ }^{\star}$ \\ ${ }^{\star}$ The Sir Alan Parks Department of Physiology, St Marks Hospital and \\ Academic Institute, Harrow, UK. \\ †Department of Surgery and Cancer, Chelsea and Westminster \\ Hospital, Fulham Road, London, UK. \\ E-mail: fareed.iqbal@nhs.net
}

doi:10.1111/apt.13555
SIRS, We read the paper by Dudekula et al. ${ }^{1}$ with interest. This analysed a national data set on colectomy for constipated patients. There has been a doubling in colectomy rates despite frequent complications, repeated hospitalisation and poor functional outcome. The authors rightfully question the benefit of colectomy for this disorder, despite increasing demand. As a national referral centre for functional bowel disorders in the UK, we have also noticed this increased demand for colectomy. Mindful of the poor outcome from colectomy, first 